http://jmscr.igmpublication.org/home/ ISSN (e)-2347-176x ISSN (p) 2455-0450

crossref DOI: https://dx.doi.org/10.18535/jmscr/v8i2.79

\title{
A Descriptive Study: Electrocardiographic and Echocardiographic Profile of Dilated Cardiomyopathy in Rajarajeswari Hospital (Cardiology Department)
}

\author{
Authors \\ Dr Rajiv Girdhar ${ }^{1}$, Dr Aashish Dilip Rayapati ${ }^{2}$, Dr Abhishek L Loonavat ${ }^{3}$ \\ ${ }^{1}$ Assistant Professor in Cardiology Department \\ ${ }^{2,3}$ Intern in RRMCH
}

\begin{abstract}
Background: Dilated cardiomyopathy (DCM) is a syndrome characterized by cardiac enlargement and impaired systolic function of one or both ventricles. Due to increased awareness of this condition along with improvement in diagnostic techniques, dilated cardiomyopathy is being recognized as a significant cause of morbidity and mortality.

Aim: Understanding the electrocardiographic and echocardiographic profile of DCM patients coming to Rajarajeswari hospital in bangalore.

Material and Methods: A descriptive study was conducted among 101 patients with dilated cardiomyopathy from the year Nov 2017 to Nov 2019. ECG and 2D Echocardiography was done among all these patients using standard techniques.

Results: Among the 101 study participants having DCM, almost all patients showed changes in ECG and $2 D$ Echocardiography. The most common ECG changes observed were ST-T changes (93\%) followed by sinus tachycardia (63.36\%). The most common $2 D$ Echo findings observed were left ventricular diastolic dimension $>5.2 \mathrm{~cm}(93 \%)$ followed by left ventricular systolic dimension $>3.9 \mathrm{~cm}(90 \%)$ and reduced left ventricular contractility ( $84 \%$ ).

Conclusion: Regular echochardiographic and electrocardiographic screening of patients with dilated cardiomyopathy is very important to identify and prevent complications among them

Keywords: Dilated Cardiomyopathy, Electrocardiographic profile, Echocardiographic profile, Arrhythmias.
\end{abstract}

\section{Introduction}

Cardiomyopathy is a primary disorder of the heart muscle that causes abnormal myocardial performance and is not result of disease or dysfunction of other cardiac structures, systemic arterial hypertension and valvular stenosis or regurgitation ${ }^{1}$. World Health Organization (WHO) and American Heart Association (AHA) classified cardiomyopathies into dilated, hypertrophic and restrictive type depending upon the basic disturbance of the function ${ }^{2,3}$. Dilated cardiomyopathy (DCM) is a syndrome characterized by cardiac enlargement and impaired systolic function of one or both ventricles. With increasing awareness of this condition along with improvement in diagnostic techniques dilated cardiomyopathy is being recognized as a significant cause of morbidity and mortality. DCM is considered to be an important cause of heart failure and accounts for up to $25 \%$ 
of all cases of congestive heart failure ${ }^{4}$. The Framingham study has reported $10 \%$ annual mortality rate for subjects having congestive cardiac failure ${ }^{5}$. Due to the increasing use of electrocardiogram (ECG) and 2D echocardiography, the incidence of dilated cardiomyopathy is also showing rising trend. It is a topic of interest of physician, cardiologist, cardiac surgeons and many other group of scientists. The present study was therefore undertaken to study the electrocardiographic and echocardiographic findings in patients with dilated cardiomyopathy.

\section{Materials and Methodology}

The current study was a descriptive cross sectional study. A total of 101 patients of dilated cardiomyopathy admitted to RAJARAJESWARI MEDICAL COLLEGE, Bangalore were enrolled in the study after taking written informed consent. The study participants were recruited over a period of 2 years from NOV 2017 to NOV 2019. All patients of DCM were advised for Electrocardiogram (ECG) and two dimensional Echocardiography (2D Echo) with color doppler at the centre itself. A 2D echocardiographic evaluation was performed according to the standards of American Society of Echocardiography in all patients using a commercially available ultrasonic system (PHILIPS EPIQCVX). Objective of this study was to understand the electrocardiographic and echocardiographic profile of the patients with dilated cardiomyopathy. A detailed history was recorded in each patient. Apart from complete blood count, ESR, FBS / PPBS, renal function tests, X-ray chest (PA view), ECG and 2D Echocardiography with colour doppler was done in all patients. All the patients were treated for congestive cardiac failure by using diuretics, ACE inhibitors, cardioselective betablockers, nitrates and cardiac glycosides. In some patients anticoagulants and anti arrhythmic drugs were also used. The data thus collected were entered and analyzed in Microsoft Office Excel. The study reports proportions of the variables under study in percentages.

\section{Results}

The following section shows the results of the analysis of the ECG and 2D Echo findings 101 patients with dilated cardiomyopathy.

Table 1 ECG Changes $(n=101)$

\begin{tabular}{|l|c|}
\hline ECG Changes & CASES \\
\hline ST-T Changes & $94(93 \%)$ \\
\hline Sinus Tachycardia & $64(63.36 \%)$ \\
\hline Sinus Bradycardia & $2(1.9 \%)$ \\
\hline Q-Wave & $13(12.8 \%\}$ \\
\hline Aterial Premature Contraction & $3(2.9 \%)$ \\
\hline Ventricular Preamture Contraction & $13(12.8 \%)$ \\
\hline Rightbundle Branch Block & $12(11.8 \%$ \\
\hline Left Bundle Branch Block & $5(4.9 \%)$ \\
\hline Right Ventricular Hypertrophy & $0(0.0 \%)$ \\
\hline Left Ventricular Hypetrophy & $13(12.8 \%$ \\
\hline Right Axis Deviation & $0(0.0 \%)$ \\
\hline Left Axis Deviation & $6(5.9 \%)$ \\
\hline Tall T Waves & $0(0.0 \%)$ \\
\hline Atrial Fibrillation & $3(2.9 \%$ \\
\hline Ventricular Tahycardia & $3(2.9 \%)$ \\
\hline Supravetricular Tahycardia & $3(2.9 \%)$ \\
\hline
\end{tabular}

As shown in table 1, following ECG changes were observed among patients. ST-T changes was seen in $94(93.8 .06 \%)$, sinus tachycardia was seen in 64 (63.3\%), Q waves, ventricular premature contractions and left ventricular hypertrophy was seen in 13 (12.8\%), right bundle branch block was seen in $12(11.8 \%)$, left axis deviation was seen in $6(5.9 \%)$, left bundle branch block in 5(4.9\%), supraventricular tachycardia, ventricular tachycardia, atrial fibrillation and atrial premature contraction in $3(2.9 \%)$ and sinus bradycardia was seen in $2(1.9 \%)$ patients.

Table 2. 2D Echocardiography Findings $(n=1.01)$

\begin{tabular}{|l|c|}
\hline 2D Echo Finding & Cases \\
\hline LV Diastolic Dimension $>5.2 \mathrm{~cm}$ & $94(93.06 \%)$ \\
\hline LV Systolic Dimension $>3.9 \mathrm{~cm}$ & $91(90.09 \%)$ \\
\hline Reduced Left Ventricular Contractility & $85(84.1 \%)$ \\
\hline Mitral Regurgitation & $49(48.51 \%)$ \\
\hline Tricuspid Regurgutation & $64(63.3 \%)$ \\
\hline Aortic Regurgitation & $0(0.0 \%)$ \\
\hline Enlarged Left Atrium & $36(31.6 \%)$ \\
\hline Left Ventriculr Out Flow Obstruction & $0(0.0 \%)$ \\
\hline Ventricular Thormbus & $0(0.0 \%)$ \\
\hline Pericardial Effussion & $13(12.8 \%)$ \\
\hline Ejection Fraction & \\
\hline$<15 \%$ & $2(1.9 \%)$ \\
\hline $16-20 \%$ & $5(4.9 \%)$ \\
\hline $21-25 \%$ & $38(37.6 \%)$ \\
\hline $26-30 \%$ & $30(29.7 \%)$ \\
\hline $31-35 \%$ & $26(25.7 \%)$ \\
\hline
\end{tabular}


Table 2 shows the 2D Echo findings among the study participants. The most common 2D echo finding was left ventricular diastolic dimension > $5.2 \mathrm{~cm}$ in 94 (93\%) followed by left ventricular systolic dimension $>3.9 \mathrm{~cm}$ in 91 (90.09\%), reduced left ventricular contractility in 85 $(84.1 \%)$, tricuspid regurgitation in $64(63.3 \%)$, mitral regurgitation in $49(48.5 \%)$, enlarged left atrium in $36(35.6 \%)$ and pericardial effusion in $13(12.8 \%)$. Aortic regurgitation, left ventricular outflow obstruction and ventricular thrombus was seen in none of the patients. Ejection fraction was observed in $38(37.6 \%)$ in range of $21-25 \%$, followed by $30(29.7 \%)$ in range of $26-30 \%$ and $26(25.7 \%)$ in range of $31-35 \%$

\section{Discussion}

The current study observed the various electrocardiographic and echocardiographic changes among patients with dilated cardiomyopathy. The ECG in patients with DCM may be remarkably normal, but abnormalities ranging from isolated $\mathrm{T}$ wave changes to septal $\mathrm{Q}$ waves in patients with extensive left ventricular fibrosis, prolongation of atrioventricular (AV) conduction, and bundle branch block may be observed. In the current study, the most common ECG changes were ST-T changes observed in almost $93 \%$ cases followed by sinus tachycardia in $63 \%$ cases. Similar findings were also reported by Yadav et al and Sachin et al in their respective studies. ${ }^{5-6}$ A study by Yadav et al. in 2003 have also reported ECG changes of bifasicular block $(\mathrm{RBBB}+\mathrm{LAHB})(10 \%)$, unifasicular block (LAHB) (16.8\%) and complete heart block (1.6\%). (5) While in our study Q waves, ventricular premature contractions and left ventricular hypertrophy was seen in $12.8 \%$, right bundle branch block was seen in $11.8 \%$, left axis deviation was seen in $5.9 \%$, left bundle branch block in $4.5 \%$, supraventricular tachycardia, ventricular tachycardia, atrial fibrillation and atrial premature contraction in $2.9 \%$ and sinus bradycardia was seen in $21.9 \%$ patients. A little bit different ECG findings were observed by
Ganesh et al in Telangana, in which left axis deviation (70\%), sinus tachycardia (48\%), ventricular premature complexes (32\%), ST-T changes (34\%) atrial fibrillation (12\%) and LBBB (20\%) were seen among DCM patients.7 While a study by Patil et al showed that the most common electocardiographic findings were LBBB, VPCs and QS pattern. ${ }^{8} 2$ D Electrocardiographic observation during the study period revealed different types of non sustained arrhythmias and conduction disturbances together with chamber enlargement as shown in Table 2. In the present study majority (38\%) of the patients had Ejection fraction (EF) of less than $30 \%$ while tricuspid regurgitation in $63 \%$ and mitral regurgitation was observed in $48 \%$ of patients. These 2D echo findings are in line with studies conducted by Singh et al and Jain et al. ${ }^{9-10}$ A dilated and poorly contracting ventricle even in sinus rhythm predisposes to thrombus formation. In our study ventricular clot/thrombus was not observed in any patients. Compared to this, study by Ganesh et al found LV clot in 4\% DCM patients and Patil et al in $19 \%$ patients. ${ }^{9-10}$ In this study, enlarged left atrium in $36(35.6 \%)$ and pericardial effusion in $13(12.8 \%)$ patients of DCM. Similar finding was observed by Ganesh et al where $30 \%$ patients of DCM showed enlarged left atrium and $8 \%$ cases showed pericardial effusion. ${ }^{9}$ Hence, such regular echochardiographic screening of patients with dilated cardiomyopathy is very important to identify and prevent complications among them.

\section{Conclusion}

The most common ECG changes observed among 101 patients of DCM were ST-T changes $(93.06 \%)$ followed by sinus tachycardia (63\%). The most common 2D Echo findings observed were left ventricular diastolic dimension $>5.2 \mathrm{~cm}$ $(94 \%)$ followed by left ventricular systolic dimension $>3.9 \mathrm{~cm}(90 \%)$ and reduced left ventricular contractility $(84 \%)$. 


\section{References}

1. Zipes D, Libby P, Bonow R, Braunwald E. A Braunwald's heart disease -Textbook of Cardiovascular Medicine, Dilated cardiomyopathy, 9th Ed. Philadelphia. Elsivier Saunders; 2012. P1563-69.

2. Richardson $\mathrm{P}, \mathrm{McKenna} \mathrm{W}$, Bristow $\mathrm{M}$, Maisch B, Mautner B, O'Connell J, et al. Report of the 1995 WHO / International Society and Federation of Cardiology (ISFC) Task Force on the Definition and Classification of cardiomyopathies. Circulation1995; 93:841-2.

3. Maron BJ, Towbin JA, Thiene G, Antzelevitch C, Corrado D, Arnett D, et al. Contemporary definitions and classification of the cardiomyopathies. Circulation 2006; 113:1807-16.

4. Anderson KM, Kannel WB. Prevalence of congestive heart failure in Framingham Heart study subjects. Circulation 1994; 13:S107-S112.

5. Yadav NA, Raghu K, LSR Krishna, Gouthami V, Jyotsana M, Seshagiri Rao D et al. Clinical Profile of Dilated Cardiomyopathy: A Tertiary Care Center Study. Indian Heart J. 2003; 55:165-178.

6. Sachin C, Phadnis P, Kalagate S. Clinical Profile of patients with dilated cardiomyopathy. JAPI 2007;23:67-74

7. Ganesh N, Rampure DM, Rajashekarappa. Etiological Study of Dilated Cardiomyopathy In a tertiary Care Hospital. Journal of Pharmaceutical and Biomedical sciences 2014; 04(10):910-13

8. Patil VC, Desai N, Galande C. Clinical and echocardiogram profile of Cardiomyopathy at tertiary care centre. Journal of Cardiovascular Disease Research 2014;5(1):34- 43.
9. Singh G, Arora P, Nayyar SB, Arora JS, Bal BS. A study of clinical profile of 124 cases of dilated cardiomyopathy. Indian Heart J 2001;72:652-658.

10. Jain A, Tewari S, Kapoor A, Kumar S, Garg N, Goel PK et al. Clinical Profile of Dilated Cardiomyopathy. Indian Heart J 2004;56:374-382. 